\title{
Bipedal Walking and Running with Compliant Legs
}

\author{
Fumiya Iida, Jürgen Rummel and André Seyfarth
}

\begin{abstract}
Passive dynamics plays an important role in legged locomotion of the biological systems. The use of passive dynamics provides a number of advantages in legged locomotion such as energy efficiency, self-stabilization against disturbances, and generating gait patterns and behavioral diversity. Inspired from the theoretical and experimental studies in biomechanics, this paper presents a novel bipedal locomotion model for walking and running behavior which uses compliant legs. This model consists of three-segment legs, two servomotors, and four passive joints that are constrained by eight tension springs. The self-organization of two gait patterns (walking and running) is demonstrated in simulation and in a real-world robot. The analysis of joint kinematics and ground reaction force explains how a minimalistic control architecture can exploit the particular leg design for generating different gait patterns. Moreover, it is shown how the proposed model can be extended for controlling locomotion velocity and gait patterns with the simplest control architecture.
\end{abstract}

\section{INTRODUCTION}

There has been an increasing interest in the use of passive dynamics for the purpose of legged locomotion. As a series of Passive Dynamic Walkers (PDWs) have demonstrated natural body dynamics for walking behavior, the minimalistic design strategy provides a few essential advantages for autonomous legged robots, i.e. simple control and energy efficiency. Previously, these robots have shown natural walking behavior on the slope [1], [2], on level ground with little actuation [3], [4], and the real-time behavior learning by exploiting the physical constraints of the morphological design and its interaction with the environment [4], [5].

However, human locomotion behavior generally exhibits more complex dynamics than those of PDWs as shown in Fig. 1 and 2. For example, the ground reaction force is very different when the system is walking or running; the joint angles of knee and ankle exhibit a few peaks in a step cycle; and the leg-ground interactions do not directly influence the fluctuation of vertical body excursion. In order to achieve adaptive bipedal locomotion, such a dynamic behavior of the legs is important for a number of reasons. The knee flexion at the beginning of touchdown, for example, would help reducing critical impacts induced by the ground reaction force, and the heel up at the end of stance phase initiates large leg swing for ground clearance. In order to achieve such

F. Iida is with Computer Science and Artificial Intelligence Laboratory, Massachusetts Institute of Technology, 32 Vassar Street, Cambridge, MA 02139, USA, and Artificial Intelligence Laboratory, Department of Informatics, University of Zurich, Andreasstrasse 15, CH-8050 Zurich, Switzerland. iida@csail.mit.edu

J. Rummel and A. Seyfarth are with Locomotion Laboratory, University of Jena, Dornburger Strasse 23, D-07743 Jena, Germany. [juergen.rummel, andre.seyfarth]@uni-jena.de

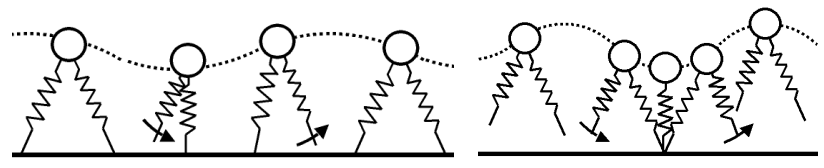

(a)

(b)

Fig. 1. Schematic illustration of the spring-mass (a) walking and (b) running. The behavior of this theoretical model matches well to human locomotion (see Fig. 2, [13]).

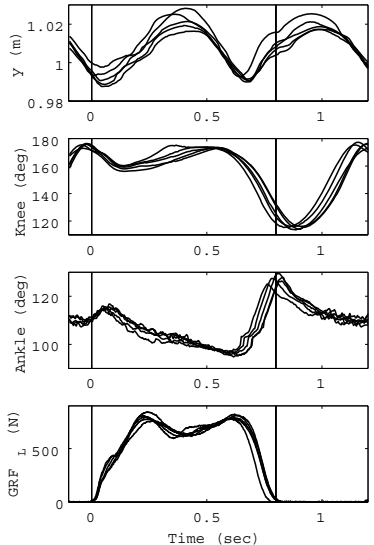

(a)
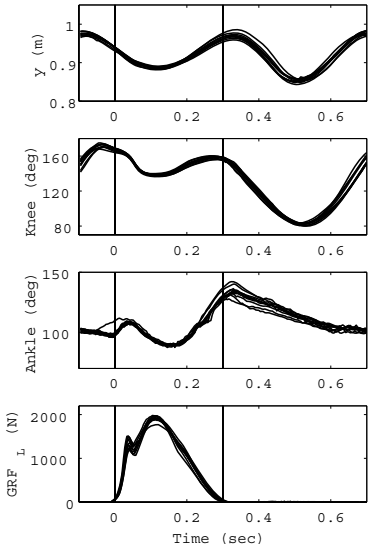

(b)
Fig. 2. Time-series trajectories of human (a) walking and (b) running. Vertical movement of body, knee joint angle, ankle joint angle, and vertical ground reaction force (from top to bottom figures) are aligned by the stance phase of 10 steps (the stance phase is indicated by two vertical lines in the figures).

a complex dynamic behavior in real time with a minimum amount of control effort, it is necessary to exploit mechanical body dynamics.

The robotics and biomechanics literature on biped locomotion reported a number of studies explaining the roles of spring-damper elements (e.g. [6], [7], [8], [9]). Among other results, investigations in biomechanics have shown that a theoretical model (the so-called spring-mass model) exhibits considerably similar bipedal running and walking behaviors to those of human [10], [11], [12], [13]. Despite its simplicity (the model consists of a point mass and a linear spring), an important implication of this line of studies is that a human seems to rely significantly on the dynamics derived from compliant legs for both running and walking behaviors.

The goal of this paper is to propose a more biologically plausible model of bipedal locomotion for both understand- 


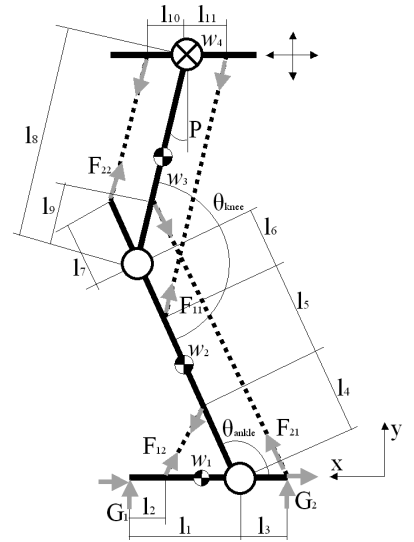

Fig. 3. Biped robot model with compliant legs investigated in this paper (only one of the two legs is shown in this figure). The model consists of a joint controlled by a motor (denoted by a circle with a cross) and three leg segments which are connected through two passive joints (white circles). The dashed lines represent the tension springs, and two ground contact points are defined in the foot segment.

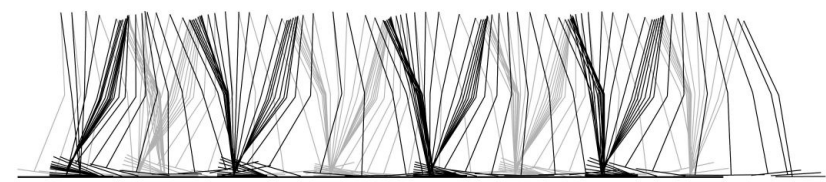

(a)

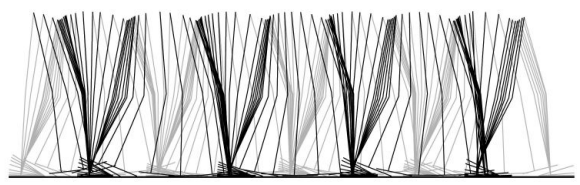

(b)

Fig. 4. (a) Walking and (b) running behavior of the simulated robot. A few typical steps of the experimental results are illustrated by stick figures.

ing human behavior and building autonomous robots. In particular, we focus on the design principles of behavioral diversity by exploiting passive dynamics as it appears to be essential in nature. This paper shows that, by implementing four tension springs in a leg with passive joints, the robot is able to self-organize itself into, at least, two stable gait patterns with a basic oscillatory actuation of the hip joints. We will first analyze the proposed model in simulation and then in the real world. As it becomes clear later in this paper, the experimental results of the proposed model show how two gait patterns of biped locomotion can be generated through a single model, which leads to further implications and research directions of biped locomotion study.

\section{BIPED MODEL WITH COMPLIANT LEGS}

\section{A. Biped Robot Model for Running and Walking}

The biped robot model consists of seven body segments (three segments in each leg and one body segment), two motors at the hip joints, four passive joints in the knee and ankle joints, and eight linear tension springs (Fig. 3). Two ground contact points are defined in each foot segment. The location of springs are inspired from the design of human legs, which is a unique feature of this robot. We conducted a heuristic search of spring configuration that is able, on the one hand, to constrain the passive joints for natural locomotion behavior, and on the other, to support the body weight of the entire system. As a result, we found that six of the springs (i.e. four springs attached between the hip and the shank, two are between the thigh and the heel) should be connected over two joints. These springs are referred to as biarticular muscles in human legs, corresponding to Rectus Femoris, Gastrocnemius, and Biceps Femoris. Another set of springs between the shank and the foot segments correspond to the monoarticular muscles, Tibialis Anterior. Eventually, the model parameters of morphological design are described as $\mathbf{M}=\left[\begin{array}{lll}\mathbf{L} & \mathbf{W} & \mathbf{S}\end{array}\right]$, which consists of the parameters of segment length $\mathbf{L}=\left[l_{1} \cdots l_{11}\right]$, and the weight parameters $\mathbf{W}=\left[\begin{array}{llll}w_{1} & w_{2} & w_{3} & w_{4}\end{array}\right]$. We defined the springs with three sets of parameters (spring constant $K_{i j}$, intrinsic damping factor $D_{i j}$, and natural length $N_{i j}$ ) as follows:

$$
\begin{aligned}
\mathbf{S} & =\left[\begin{array}{llll}
\mathbf{S}_{\mathbf{1 1}} & \mathbf{S}_{\mathbf{1 2}} & \mathbf{S}_{\mathbf{2 1}} & \mathbf{S}_{\mathbf{2 2}}
\end{array}\right] \\
& =\left[\begin{array}{llll}
K_{11} & K_{12} & K_{21} & K_{22} \\
D_{11} & D_{12} & D_{21} & D_{22} \\
N_{11} & N_{12} & N_{21} & N_{22}
\end{array}\right]
\end{aligned}
$$

The force generated in these tension springs are calculated as:

$$
F_{i j}= \begin{cases}K_{i j}\left(x_{i j}-N_{i j}\right)-D_{i j} \dot{x}_{i j} & : x_{i j}>0 \\ 0 & : x_{i j} \leq 0\end{cases}
$$

where $x_{i j}$ denotes the length of the spring.

This model requires only three control parameters of the motors: $\mathbf{C}=\left[\begin{array}{lll}A & B & \omega\end{array}\right]$, amplitude, offset angle, and frequency, respectively. These parameters determines a simple oscillation of the hip motors, in which the angular positions of the hip joints are determined by the following sinusoidal curve:

$$
\begin{aligned}
P_{r}(t) & =A \sin (\omega t)+B \\
P_{l}(t) & =A \sin (\omega t+\pi)+B
\end{aligned}
$$

To facilitate the real-world implementation for the robotic experiments, the dimension of this model is scaled down as shown in Appendix section. And for the sake of simplicity, this model is restricted to motion within a plane, thus no rotational movement (roll or pitch) of the body segment is considered. In the following simulation and robot experiments, all of the parameters $\mathbf{S}$ and $\mathbf{C}$ were heuristically determined.

\section{B. Body Dynamics of Walking and Running}

This model was implemented and analyzed in Matlab simulation together with the SimMechanics toolbox. In the simulation, we evaluated the model on a level ground surface with a physically realistic interaction model based on a 

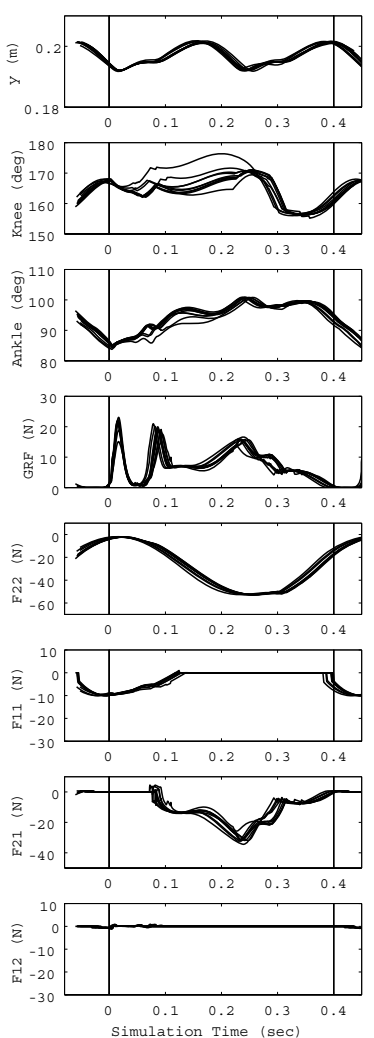

(a)

(b)

Fig. 5. Time-series trajectories of (a) walking and (b) running behavior of the simulated robot. Vertical movement of the body, angular trajectories of knee and ankle joints, vertical ground reaction forces, and the forces $F_{22}, F_{11}, F_{21}$, and $F_{12}$ generated in springs $S_{22}, S_{11}, S_{21}$, and $S_{12}$, respectively. The experimental data of 10 steps are aligned with respect to the initial peak of each stance phase (indicated by the vertical lines).

biomechanical study [14]. The vertical ground reaction forces are approximated by nonlinear spring-damper interaction, and the horizontal forces are calculated by a sliding-stiction model. The model switches between sliding and stiction when the velocity of the foot becomes lower or higher than the specified limit determined by the sliding and stiction friction coefficients, $\mu_{\text {slide }}$ and $\mu_{\text {stick}}$, respectively.

$$
\begin{aligned}
G_{y i} & =-2.5 e^{5} \cdot y_{c i}^{3}\left(1-3.3 \dot{y}_{c i}\right) \\
G_{x i} & = \begin{cases}\mu_{s l i d e} \dot{x}_{c i} G_{y i} & \mu_{\text {slide }} \dot{x}_{c i} G_{y i}>\mu_{\text {stick }} G_{y i} \\
F_{x c i} & \mu_{\text {slide }} \dot{x}_{c i} G_{y i j} \leq \mu_{\text {stick }} G_{y i}\end{cases}
\end{aligned}
$$

where $\dot{x}_{c i}$ and $y_{c i}$ denote the horizontal velocity and the non-dimensional vertical distance of the contact point $i$ from the ground surface, respectively. $F_{x c i}$ represents the force required to prevent the contact point sliding.

To describe the dynamics of the proposed model, in the following analysis, we evaluate the time-series data of state variables $\mathbf{V}=\left[\begin{array}{lllll}y & \theta_{\text {knee }} & \theta_{\text {ankle }} & G_{y 1}+G_{y 2} & \mathbf{F}\end{array}\right]$, i.e.
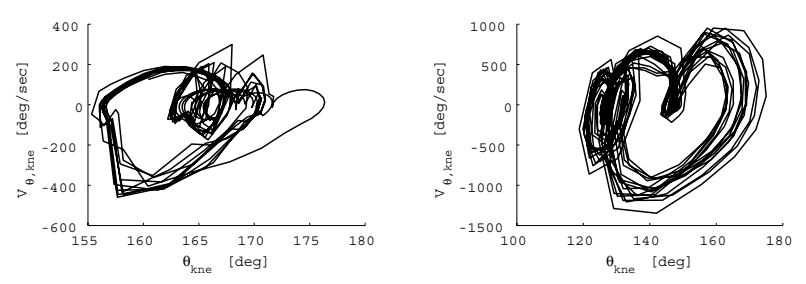

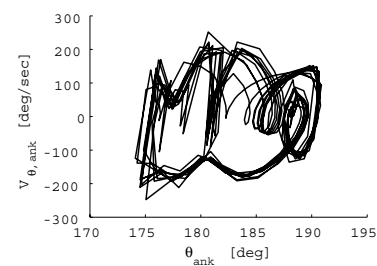

(a)

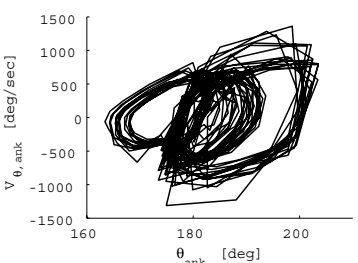

(b)
Fig. 6. Phase plot of knee and ankle joints during (a) walking and (b) running behavior of the simulated robot.

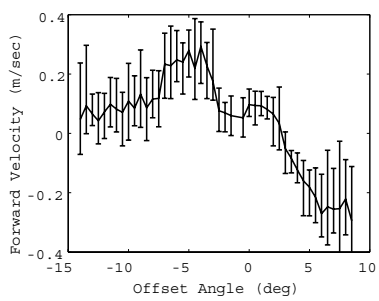

(a)

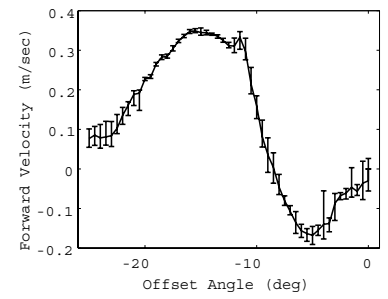

(b)
Fig. 7. Forward velocity of (a) walking and (b) running behavior in simulation with respect to the offset angle of the motor oscillation. The vertical line in each plot represents the variance of forward speed in 10 experiments, each of which was tested with different coefficients of ground friction.

vertical position of the body segment, knee and ankle joint angles, ground reaction force of a $\operatorname{leg}^{1}$, and a vector of forces generated in the springs.

With the spring and control parameters $\mathbf{S}_{\text {walk }}$ and $\mathbf{C}_{\text {walk }}$ (see Appendix section), the model exhibits stable walking gait as shown in Fig. 4(a) and Fig. 5(a). The behavior of each joint shows the similarity to those of human walking (Fig. 2(a)). More specifically, in Fig. 4(a), the knee joint starts slightly flexing at the beginning of stance phase, extending and flexing again toward the end. Also, the ankle joint extends toward the swing phase resulting in heel up.

When the spring and control parameters are set to $\mathbf{S}_{\text {run }}$ and $\mathbf{C}_{\mathbf{r u n}}$, the behavior of this model is changed into a running gait with clear flight phases (Fig. 4(b) and 5(b)). By comparing the simulation and human (Fig. 2(b) and Fig. 5(b)), the knee and ankle joints show similar behavioral patterns. For example, the lowest peak of the body vertical excursion occurs at the middle of stance phase; the knee joint exhibits multiple peaks in a cycle; and the ankle joint extends both at the beginning of stance phase and in the flight phase.

\footnotetext{
${ }^{1}$ The ground reaction force of one leg is a sum of two contact points of the foot (see also Fig. 3).
} 


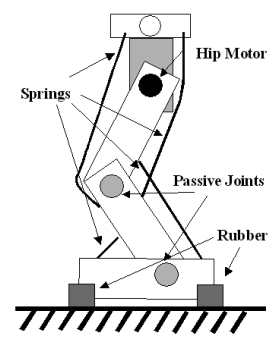

(a)

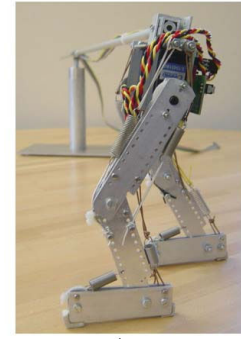

(b)

Fig. 8. (a) Schematic illustration and (b) photograph of the biped robot. This robot consists of a joint controlled by a servomotor (represented by a black circle) and three leg segments which are connected through two passive joints (gray circles). Four tension springs are attached to the segments and rubber materials are implemented at the two ground contact points of the foot segment.

It is important to mention that this model is able to achieve these two gaits with significantly different dynamics by simply changing the parameters of springs and motor control. The different dynamics are shown in the time-series data of the ground reaction force and the spring activation (Fig. 5). The ground reaction force shows multiple humps during walking while there is a large bell curve in running, which is similar to the data in human experiments (Fig. 2).

The phase plot of the knee and ankle joints shows clearer structure of the gait patterns (Fig. 6). These figures show in which state of the cycle the behavior of the legs is stabilized. For example, the variance of the knee joint trajectories is smaller at the higher angular velocity (i.e. the upper left side of the figures). This means that the gait is more stabilized at the particular period in a cycle, i.e. at the end of stance phase in the walking gait, and at the beginning of stance phase in the running gait. Furthermore, by interpreting the phase plots Fig. 6 together with the time series Fig. 5, we can understand which springs contribute to the stabilization. Namely, for the walking gait, $S_{22}$ and $S_{21}$ are active at the end of stance phase, while for the running gait, $S_{22}, S_{11}$ and $S_{21}$ are active at the beginning of stance phase.

\section{Stability and Forward Velocity}

When one of the motor control parameters is changed, it influences the forward velocity of locomotion. By exploiting these characteristics, the proposed model is able to control the locomotion velocity in a simple manner. In this subsection, we explore how the forward velocity is influenced by the offset angle of motor oscillation (i.e. $B$ in Equations (3) and (4)). In addition, by varying the coefficients of the ground friction, we evaluate the locomotion stability of the proposed model.

In the following experiments, we repeated the abovementioned walking and running simulation experiments by using the different parameters: for walking experiments, the motor offset angle $B$ was set to between -12 and 8 degrees by 0.5 degree step, and for running experiments, between 25 and 5 degrees also by 0.5 degree step. The mean forward velocity during the ten second experiment was measured in
TABLE I

SPECIFICATION OF THE ROBOT.

\begin{tabular}{|c||c|c|}
\hline Param. & Description & Value \\
\hline \hline$l_{8}$ & Thigh & $0.10 \mathrm{~m}$ \\
\hline$l_{4}+l_{5}+l_{6}$ & Shank & $0.10 \mathrm{~m}$ \\
\hline$l_{1}$ & Foot & $0.04 \mathrm{~m}$ \\
\hline$l_{4}, l_{6}, l_{7}, l_{9}$ & Spring Attach. & $0.02 \mathrm{~m}$ \\
\hline$l_{3}$ & Heel & $0.02 \mathrm{~m}$ \\
\hline$S_{11}$ & Spring const. & $1.8 N / \mathrm{mm}$ \\
\hline$S_{12}$ & Spring const. & $0.4 N / \mathrm{mm}$ \\
\hline$S_{21}$ & Spring const. & $1.8 N / \mathrm{mm}$ \\
\hline$S_{22}$ & Spring const. & $1.3 \mathrm{~N} / \mathrm{mm}$ \\
\hline$M$ & Total mass & $0.95 \mathrm{~kg}$ \\
\hline
\end{tabular}

ten different coefficients of ground friction $\left(0.65<\mu_{\text {stick }}<\right.$ 0.85 and $0.45<\mu_{\text {slide }}<0.65$ ). Fig. 7 shows the mean forward velocity of ten experiments and variance induced by the different ground friction.

In both walking and running, it is clearly shown that the forward velocity is significantly influenced by the offset angle, and there exists a preferred offset angle with which the system gains the maximum velocity. From Fig. 7, the forward velocity of the dynamic biped walking and running can be in principle controlled by simply varying the offset angle of motor oscillation. It is also important to note that the similar changes of forward velocity were observed by using the other parameters of frequency and amplitude (i.e. $A$ and $\omega$ in Equations (3) and (4)).

We can also observe stability of locomotion behavior in these figures by evaluating the variance of forward locomotion speed induced by a different set of coefficients of ground friction. In general, the running gait has less variance against different ground friction than that of walking, which indicates the running gait is more stable. In particular, it exhibits more stable behavior at the preferred velocity. From our observation of the simulation experiments, the stability mostly depends on how the system interacts with the ground: in running at the preferred velocity, the system interacts with the ground in a simpler manner by touching down only with the toes, for example.

\section{ROBOT EXPERIMENTS}

The proposed model was subsequently implemented in a physical robot platform as shown in Fig. 8. This robot consists of passive joints in knees and ankles, and two commercial servomotors (Conrad HS-9454) are used in the hip joints as in the simulation model. We used four tension springs and rubber material at the two ground contact points in each foot segment in order to gain large ground friction and to minimize impact force at touch down. A supporting boom was attached to the body segment in order to restrict the roll and pitch movement of the body segment. The same control parameters were used to conduct a set of 


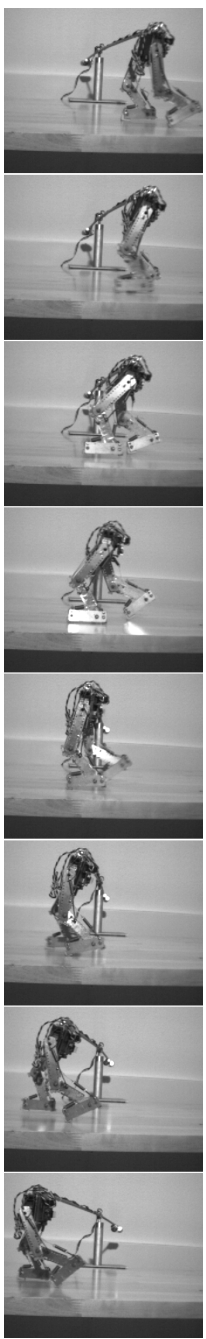

(a)

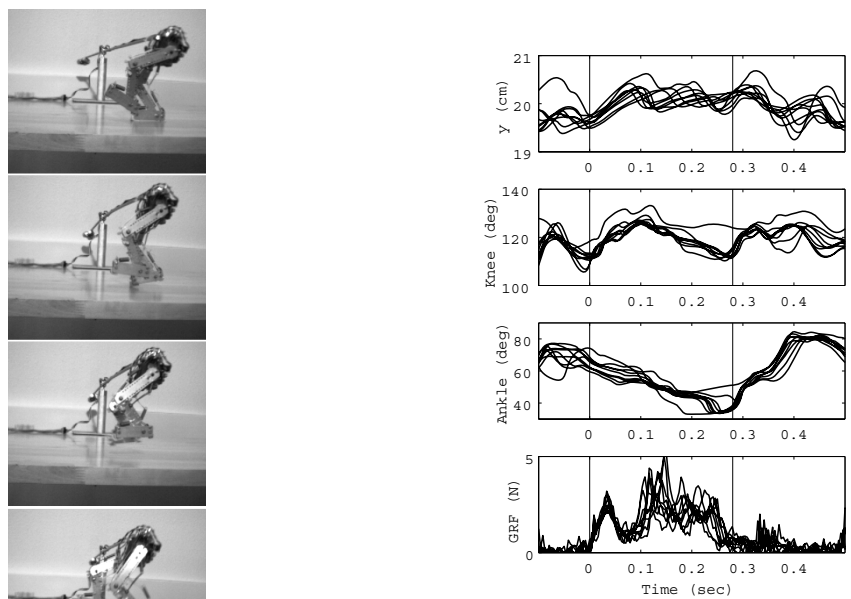

(a)
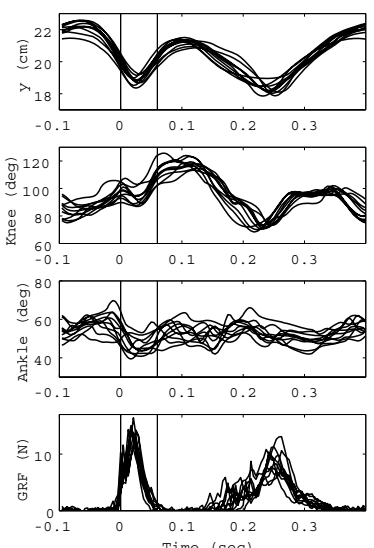

(b)
Fig. 10. Time-series trajectories of the real-world robot (a) walking and (b) running. Vertical movement of body, knee joint angle, ankle joint angle, and vertical ground reaction force (from top to bottom figures) are aligned by the stance phase of 10 steps (the stance phase is indicated by two vertical lines in the figures).

were then filtered and aligned with respect to the ground reaction force. In general, the experimental results show a good agreement with the simulation results: the vertical excursion of the body is larger in running than in walking; the overall movement of joint angles are similar; and the ground reaction force shows multiple peaks in walking while there is a single peak in running ${ }^{4}$.

It is important to mention that, with more precise observation, the biped robot could take advantage of a few unique characteristics of the joint trajectories in the proposed model. For example, the flexion of the knee joint at the beginning of touch down would help absorbing both intensive ground reaction force and deviations derived from the ground surface, by exploiting the elastic properties of the knee joint.

\section{DISCUSSION AND CONCLUSION}

experiments ${ }^{2}$.

Fig. 9 shows time-series photographs of a typical locomotion behavior recorded by a high-speed camera (Basler A602fc: resolution $656 \times 490$ pixels, frame rate $100 \mathrm{fps})^{3}$. It can be clearly observed in the walking gait (Fig. 9(a)) that the knee joint is extended at the take off of the left leg, and that the ankle joint is flexed. And at the beginning of touchdown, the knee joint is bent.

More detailed behavior is shown in the kinematics and ground reaction force measured during ten steps of the robot walking and running by a set of high-speed infrared cameras for motion capture (six Qualisys motion capture units; sampling frequency of $240 \mathrm{~Hz}$ ) and force plates (Kistler 9281B11; sampling frequency of $1000 \mathrm{~Hz}$ ). The data

\footnotetext{
${ }^{2}$ Since this robot is not able to change the spring parameters, we tuned the parameters before each experiment. These parameters were again determined by a heuristic search.

${ }^{3}$ See also the video clip.
}

Since the Central Pattern Generator circuits have discovered in biological systems, there has been an on-going debate on the control mechanisms of legged locomotion. In particular, the degree to which the mechanical body dynamics contributes to the stability, energy efficiency, and behavioral diversity of legged locomotion processes is not clear. Toward our further understanding of walking and running dynamics in robots and biological systems, this paper presented a novel model of bipedal locomotion with a special focus on the mechanical constraints (i.e. the elastic legs with passive joints). A set of experimental results in simulation and a robotic platform has shown that, by having a unique mechanical structure, two stable locomotion patterns can be self-organized through the dynamic system-environment interactions. Furthermore, it is shown that, forward locomotion

\footnotetext{
${ }^{4}$ In the running experiments, both of the legs are on the force plate. Therefore the second peak of the ground reaction force in Fig. 10(b) indicates the force generated by the other leg.
} 
velocity and gait patterns can be controlled by extending this control framework. The significance of this approach is the fact that human-like bipedal locomotion can be controlled by using a very simple control architecture: it can potentially use a simple sinusoidal oscillation of hip actuation.

There are a few important implications derived from the experimental results presented in this paper. Firstly, although we have searched through the morphological and control parameters only in a heuristic manner, it will be necessary to conduct a systematic optimization of these parameters to more thoroughly understand the proposed model. Along the similar lines, an adaptive and learning function of motor control is a prerequisite for the proposed model. In more complex environments with various ground conditions, for example, the proposed model will be required to adaptively adjust the control parameters, as shown partially in Fig. 7. Moreover, another significant challenge will be the extension to more demanding constraints of morphological properties. It is not clear, for example, how the model could cope with the rotational movement of the body segment (i.e. removing the supporting boom of the robot). It might be required to implement an upper body to compensate for these rotational movements.

So far we have explored only a small part of all behavioral variations. Considering the fact that we explored only a few sets of the parameters, we expect that many more behavioral variations could be possible within the proposed framework. It is particularly interesting to investigate whether there is a set of parameters which requires only a little change in the spring parameters for two different gaits, for example. The capability to change the spring parameters $\mathbf{S}$ seems to be another important prerequisite for more biologically plausible and adaptive legged locomotion. Because of the absence of stiffness control in the proposed model, many behavioral patterns of humans are not possible. For example, the foot steps in the running behavior of our experiments are relatively short compared with that of walking, which results in a relatively slow running speed (see Fig. 4, 7 and the associated video clip). As a result, this running behavior is more similar to a slow jogging or hopping of human locomotion, rather than running. It seems that, for larger running speed and more human-like running behavior, some of the springs need to be able to change their stiffness in a step cycle, which must be investigated further in the future.

\section{ACKNOWLEDGMENTS}

This work is supported by the German Research Foundation (DFG, SE1042), the Swiss National Science Foundation (Grant No. 200021-109210/1), and the Swiss National Science Foundation Fellowship for Prospective Researchers (Grant No. PBZH2-114461). The authors would like to appreciate Susanne Lipfert and our other collaborators for providing us the experimental results of human locomotion.

\section{REFERENCES}

[1] McGeer, T. (1990). Passive dynamic walking, The International Journal of Robotics Research, Vol. 9, No. 2, 62-82.
[2] Collins, S. H., Wisse, M., and Ruina, A. (2001). A three-dimentional passive-dynamic walking robot with two legs and knees. International Journal of Robotics Research 20, 607-615.

[3] Wisse, M. and van Frankenhuyzen, J. (2003). Design and construction of MIKE: A 2D autonomous biped based on passive dynamic walking. Proceedings of International Symposium of Adaptive Motion and Animals and Machines (AMAMO3).

[4] Collins, S., Ruina, A., Tedrake, R., and Wisse, M. (2005). Efficient bipedal robots based on passive dynamic walkers, Science Magazine, Vol. 307, 1082-1085.

[5] Tedrake, R., Zhang, T.W. and Seung, H.S. (2004). Stochastic policy gradient reinforcement learning on a simple 3D biped. Proc. of the 10th Int. Conf. on Intelligent Robots and Systems, 3333-3334.

[6] Srinivasan, M., and Ruina, A. (2006). Computer optimization of a minimal biped model discovers walking and running, Nature, Vol. 439, No. $5,72-75$

[7] Jalics, L., Hemami, H., and Clymer, B. (1997). A control strategy for terrain adaptive bipedal locomotion, Autonomous Robots, Vol. 4, No. 3, 243-257.

[8] McMahon, T. A. (1984). Muscles, reflexes, and locomotion, Princeton University Press.

[9] Alexander, R. McN. (1997). A model of bipedal locomotion on compliant legs, Phil. Trans. R. Soc. Lond. B 338, 189-198.

[10] McMahon, T. A., Cheng, G. C. (1990). The mechanics of running: How does stiffness couple with speed?, J. Biomechanics, Vol. 23, Suppl. 1, 65-78.

[11] Blickhan, R. (1989). The spring-mass model for running and hopping. J. Biomech. 22, 1217-1227.

[12] Seyfarth, A., Geyer, H., Guenther, M., Blickhan, R. (2002). A movement criterion for running, Journal of Biomechanics, 35, 649655 .

[13] Geyer H, Seyfarth A, Blickhan R. (2006). Compliant leg behaviour explains basic dynamics of walking and running. Proc. Roy. Soc. Lond. B. DOI 10.1098/rspb.2006.3637, 1471-2954.

[14] Gerritsen, K. G. M., van den Bogert, A. J., and Nigg, B. M. (1995). Direct dynamics simulation of the impact phase in heel-toe running, Journal of Biomechanics, Vol. 28, No. 6, 661-668.

\section{APPENDIX}

The parameters used in the experiments:

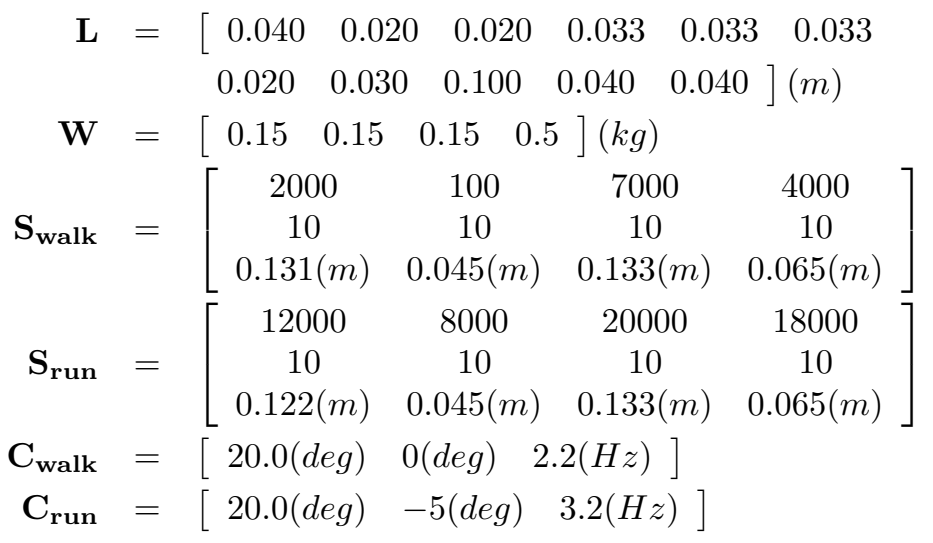

